\title{
Peripheral T-cell lymphoma, not otherwise specified: a report of 340 cases from the International Peripheral T-cell Lymphoma Project
}

\author{
Dennis D. Weisenburger, ${ }^{1}$ Kerry J. Savage,${ }^{2}$ Nancy Lee Harris, ${ }^{3}$ Randy D. Gascoyne, ${ }^{4}$ Elaine S. Jaffe,${ }^{5}$ \\ Kenneth A. MacLennan, ${ }^{6}$ Thomas Rüdiger, ${ }^{7}$ Stefano Pileri, ${ }^{8}$ Shigeo Nakamura, ${ }^{9}$ Bharat Nathwani, ${ }^{10}$ Elias Campo, ${ }^{11}$ \\ Francoise Berger, ${ }^{12}$ Bertrand Coiffier, ${ }^{13}$ Won-Seog Kim, ${ }^{14}$ Harald Holte, ${ }^{15}$ Massimo Federico, ${ }^{16}$ Wing Y. Au, ${ }^{17}$ \\ Kensei Tobinai, ${ }^{18}$ James O. Armitage, ${ }^{19}$ and Julie M. Vose, ${ }^{19}$ for the International Peripheral T-cell Lymphoma Project
}

1Department of Pathology and Microbiology, University of Nebraska Medical Center, Omaha, NE; ²Department of Medical Oncology, British Columbia Cancer Agency, Vancouver, BC; ${ }^{3}$ Department of Pathology, Massachusetts General Hospital and Harvard Medical School, Boston, MA; ${ }^{4}$ Department of Pathology, British Columbia Cancer Agency, Vancouver, BC; ${ }^{5}$ Department of Hematopathology, National Cancer Institute, Bethesda, MD; ${ }^{6}$ Section of Pathology and Leeds Institute of Molecular Medicine, St James University Hospital, Leeds, United Kingdom; ${ }^{7}$ Institute of Pathology, Städtisches Klinikum Karlsruhe, Karlsruhe, Germany; ${ }^{8}$ Department of Pathology, University of Bologna Hospital, Bologna, Italy; ${ }^{9}$ Department of Pathology and Laboratory Medicine, Nagoya University Hospital, Nagoya, Japan; ${ }^{10}$ Department of Pathology, Cedars-Sinai Medical Center and University of Southern California Keck School of Medicine, Los Angeles, CA; ${ }^{11}$ Department of Pathology, University of Barcelona Hospital Clinic, Barcelona, Spain; ${ }^{12}$ Department of Pathology, Centre Hospitalier Lyon-Sud, Lyon, France; ${ }^{13}$ Department of Medicine, Centre Hospitalier Lyon-Sud, Lyon, France; ${ }^{14}$ Division of Hematology-Oncology, Samsung Medical Center and Sungkyunkwan University Medical School, Seoul, Korea; ${ }^{15}$ Department of Oncology, Norwegian Radium Hospital and Oslo University Hospital, Oslo, Norway; ${ }^{16}$ Department of Medicine, Modena Hospital, Modena, Italy; ${ }^{17}$ Department of Medicine, Queen Mary Hospital, Hong Kong, China; ${ }^{18} \mathrm{Hematology}$ and Stem Cell Transplantation Division, National Cancer Center Hospital, Tokyo, Japan; and ${ }^{19}$ Department of Internal Medicine, University of Nebraska Medical Center, Omaha, NE

The International Peripheral T-cell Lymphoma Project is a collaborative effort to better understand peripheral T-cell lymphoma (PTCL). A total of 22 institutions submitted clinical and pathologic material on 1314 cases. One objective was to analyze the clinical and pathologic features of 340 cases of PTCL, not otherwise specified. The median age of the patients was 60 years, and the majority $(69 \%)$ presented with advanced stage disease. Most patients (87\%) presented with nodal disease, but extranodal disease was present in $62 \%$. The 5 -year overall survival was $32 \%$, and the 5 -year failure-free survival was only $20 \%$. The majority of patients $(80 \%)$ were treated with combination chemotherapy that included an anthracycline, but there was no survival advantage. The International Prognostic Index (IPI) was predictive of both overall survival and failure-free survival $(P<.001)$. Multivariate analysis of clinical and pathologic prognostic factors, respectively, when controlling for the IPI, identified bulky disease $(\geq 10 \mathrm{~cm})$, thrombocytopenia ( $\left.<150 \times 10^{9} / \mathrm{L}\right)$, and a high number of transformed tumor cells (> $70 \%$ ) as adverse predictors of survival, but only the latter was significant in final analysis. Thus, the IPI and a single pathologic feature could be used to stratify patients with PTCL-not otherwise specified for novel and risk-adapted therapies. (Blood. 2011;117(12):3402-3408)

\section{Introduction}

Peripheral T-cell lymphoma (PTCL) and natural killer/T-cell lymphoma (NKTCL) are an uncommon and heterogeneous group of disorders that compose $5 \%$ to $20 \%$ of all non-Hodgkin lymphomas (NHLs) in different parts of the world. ${ }^{1,2}$ In recent years, the incidence of PTCL and NKTCL in the United States has increased by almost 3-fold with an annual increase of $3.8 \%$, whereas the incidence of B-cell lymphoma and Hodgkin lymphoma has been relatively stable..$^{3,4}$

One of the most common subtypes of PTCL is a heterogeneous group of nodal and extranodal mature T-cell lymphomas that do not correspond to any of the specifically defined T-cell entities in the World Health Organization classification, ${ }^{1}$ and are therefore called PTCL, not otherwise specified (NOS). Uncommon variants of PTCL-NOS include lymphoepithelioid (Lennert) lymphoma, and cases with a follicular or T-zone pattern of growth. ${ }^{1}$ Over the last 12 years, several clinical studies have attempted to identify the clinical and pathologic features of prognostic importance in PTCL-NOS, but the number of cases in these studies was generally small and the findings have been inconsistent or unconfirmed. ${ }^{5-13}$

The International Peripheral T-cell Lymphoma Project was undertaken as a large retrospective study of PTCL and NKTCL in North America, Europe, and Asia with the goal of better characterizing this group of NHL. One objective of was to analyze the clinical and pathologic features of the 340 cases of PTCL-NOS in the study, and to determine the important prognostic factors for this uncommon entity.

\section{Methods}

Twenty-two institutions in North America, Europe, and Asia participated in the study (supplemental Appendix, available on the Blood Web site; see the Supplemental Materials link at the top of the online article). Approval for
Submitted September 30, 2010; accepted January 3, 2011. Prepublished online as Blood First Edition paper, January 26, 2011; DOI 10.1182/blood-2010-09310342 .

The online version of this article contains a data supplement.
The publication costs of this article were defrayed in part by page charge payment. Therefore, and solely to indicate this fact, this article is hereby marked "advertisement" in accordance with 18 USC section 1734. 
the study was obtained from the Institutional Review Board at the coordinating center (University of Nebraska Medical Center) and at each participating center per the institutional policy. The cases selected for the study were previously untreated patients 19 years of age or older with de novo PTCL or NKTCL, excluding mycosis fungoides and Sézary syndrome, who were diagnosed between January 1, 1990 and December 31, 2002. The patients were consecutive from each institution and were required to have adequate tissue biopsies for diagnosis and classification. Patients with only needle aspiration cytology specimens were excluded. At each institution, the local pathologist reviewed the diagnostic pathology slides and reports for each case, and recorded the results of local immunophenotypic, cytogenetic, and molecular genetic studies that had been performed in the initial diagnosis of the case on a standard phenotype datasheet. The local pathologist also selected representative slides and a formalin-fixed tissue block from each case to submit for regional review and more detailed immunophenotyping. Cases in which the tissue blocks were exhausted or no longer available for study were also acceptable if the slides and immunostains or flow cytometric data were available for review and adequate for diagnosis and classification. Clinical characteristics of the patients, including treatment data and follow-up information, were also required.

From each institution, the phenotype datasheets, diagnostic slides, and tissue blocks were sent to one of 5 regional centers for review and evaluation by an expert hematopathologist. These centers included Omaha, NE (D.D.W.); Leeds, United Kingdom (K.A.M.); Würzburg, Germany (T.R.); Bologna, Italy (S.P.); and Nagoya, Japan (S.N.). A standard panel of immunostains was performed on each case, including CD20, CD2, CD3, CD4, CD5, CD8, CD30, CD56, TCR- $\beta$, TIA-1, and Ki67, and in situ hybridization stains for Epstein Barr virus-encoded RNAs (EBERs). An immunostain was considered positive if more than $20 \%$ of the tumor cells stained. Other immunostains, polymerase chain reaction analyses, and fluorescence in situ hybridization cytogenetic studies were performed as needed, and all cases were diagnosed according to the criteria of the World Health Organization classification. ${ }^{1}$ The percentages of transformed tumor cells (blasts), and tumor cells expressing CD30 or Ki67, were also estimated in 5\% increments for each case, as were the percentages of background nontumor cells expressing either CD4 or CD8. The number of cells staining for EBERs was evaluated semiquantitatively by counting positive cells in the 10 most positive fields using a $10 \times$ ocular lens and a $20 \times$ objective, and calculating the average number per field (f): $0 / \mathrm{f}=0 ;<1 / \mathrm{f}=1+; 1$ to $9 / \mathrm{f}=2+; 10$ to $50 / \mathrm{f}=3+;$ and $>50 / \mathrm{f}=4+$. The results of these studies and the diagnosis were recorded by the regional expert on standard phenotype and diagnosis datasheets, respectively, for each case.

Panels of 4 expert hematopathologists, drawn from the contributing local sites and regional centers, then traveled to the regional centers to review the cases. The composition of the panels differed at the various regional centers. At each center, the diagnostic slides for each case were classified independently by each expert according to the criteria of the World Health Organization classification. ${ }^{1}$ The initial diagnosis was based on examination of the hematoxylin and eosin- and/or Giemsa-stained slides, the immunostains, and the phenotype datasheets, but with only limited clinical information from the time of initial diagnosis, including the anatomic biopsy site and the site of the largest tumor mass (ie, diagnosis 1). After recording this diagnosis, the expert was presented with the entire clinical datasheet and a second diagnosis was rendered (ie, diagnosis 2). The previous diagnosis could not be changed based on the clinical information subsequently revealed. If a case was considered unclassifiable, the expert was required to give a reason, such as inadequate material, poor slide preparation, additional immunophenotyping needed, additional information needed, or other reasons. Each expert also estimated the percentage of transformed tumor cells (blasts) in 5\% increments for each case. The median of the estimates of the 4 panel experts and the regional expert was used as the percentage of transformed cells for each case. Approximately 50 to 60 cases were reviewed by each expert each day.

In addition to the independent diagnosis rendered by each of the 4 expert hematopathologists, a consensus diagnosis was also reached in each case. A consensus was considered to have been reached if at least 3 of the 4 experts on the panel agreed on the second diagnosis (diagnosis 2). All cases without a consensus diagnosis and all unclassifiable cases were jointly reviewed on a multiheaded microscope and discussed by the 4 experts in a daily consensus conference, and an attempt was made to reach a consensus diagnosis. If additional sections, immunostains, molecular or cytogenetic studies, or other information was required, a diagnostic algorithm was developed by the panel and the additional materials or data were obtained, if possible, and reviewed at a subsequent consensus conference at the center. If the additional materials or data could not be obtained during the site visit, the required materials and information were subsequently sent to the expert hematopathologist at the regional center who arbitrated the case based on the algorithm.

The clinical information for each case was abstracted from the medical record and recorded on a standard clinical datasheet for computerized data entry. These data included coded patient and site identifiers, sex, ethnicity, date of birth, site of the diagnostic biopsy, other sites of disease, and Ann Arbor stage. Additional data recorded included the symptoms at diagnosis, site and diameter of the largest tumor mass, performance status, and a history of prior immunosuppressive therapy or immune system disorder. Laboratory data recorded included hemoglobin, platelet count, white blood cell count, presence of circulating lymphoma cells, and serology for HIV and human T-lymphotropic virus, type-1 (HTLV-1). The serum lactate dehydrogenase, $\beta_{2}$-microglobulin, and C-reactive protein levels, and the presence of hypercalcemia, hypogammaglobulinemia, hypergammaglobulinemia, monoclonal serum immunoglobulin, hemolytic anemia, and hemophagocytic syndrome were recorded. The initial therapy and response, details of remission, progression or relapse, and subsequent therapies, along with survival status and cause of death, were recorded. In some cases, sufficient data were not available for inclusion in some of the clinical or survival analyses.

Completed clinical and pathology datasheets were reviewed and edited to detect any inconsistencies, and additional information or clarification was obtained when needed. After editing, the clinical and pathology data were entered into a computer for data analysis. The International Prognostic Index (IPI) ${ }^{14}$ was used to stratify patients, and a new prognostic model for PTCL-NOS was evaluated. ${ }^{9}$ Treatment outcome was determined by overall survival (OS) and failure-free survival (FFS). OS was defined as the time from diagnosis to death from any cause, with surviving patient follow-up censored at the last contact date. FFS was defined as the time from diagnosis to the first occurrence of progression, relapse after response, or death resulting from any cause. Follow-up of patients not experiencing any of these events was censored at the date of last contact. Estimates of OS and FFS distributions were calculated using the method of Kaplan and Meier, ${ }^{15}$ and time-to-event distributions were compared using the log-rank test. ${ }^{16}$ Clinical and prognostic factor comparisons were performed using the $\chi^{2}$ or Fisher exact test. Multivariate analysis was performed with the Cox proportional hazards regression model using stepwise selection.

\section{Results}

Of the 1314 eligible cases submitted, a diagnosis of PTCL or NKTCL was made in 1153 cases $(87.8 \%)$, and 340 of these were PTCL-NOS (29.5\%). Of the latter, 101 cases (30\%) were from North America, 135 (40\%) were from Europe, and 104 (30\%) were from Asia. Among the 340 PTCL-NOS cases, 301 (88.5\%; $26.1 \%$ of all cases) were classified as unspecified PTCL, $28(8.2 \% ; 2.4 \%)$ as lymphoepithelioid (Lennert) PTCL, 6 (1.8\%; $0.5 \%)$ as follicular PTCL, and $5(1.5 \% ; 0.5 \%)$ as T-zone PTCL. The immunophenotypic features of the cases are shown in Table 1. Immunostaining for TCR- $\beta$ was not very useful because many cases of presumed $\alpha-\beta$ type failed to stain. The EBER ${ }^{+}$ cells were background small and large B cells. Lymphoepithelioid PTCL was more likely to express CD8 and TCR- $\beta$, and less 
Table 1. Immunophenotypic features of PTCL-NOS

\begin{tabular}{lcc}
\hline Immunophenotype & $\begin{array}{c}\text { PTCL-NOS, } \\
\%(\mathbf{n} / \mathbf{N})\end{array}$ & $\begin{array}{c}\text { Lymphoepithelioid PTCL, } \\
\%(\mathbf{n} / \mathrm{N})\end{array}$ \\
\hline CD2 & $86(211 / 244)$ & $100(13 / 13)$ \\
CD3E & $93(238 / 255)$ & $88(14 / 16)$ \\
CD4 & $66(140 / 213)$ & $64(9 / 14)$ \\
CD5 & $69(170 / 245)$ & $87(13 / 15)$ \\
CD8 & $19(48 / 250)$ & $44^{*}(7 / 16)$ \\
CD30 & $32(69 / 217)$ & $12^{*}(2 / 16)$ \\
CD56 & $6(13 / 219)$ & $8(1 / 13)$ \\
TCR- $\beta$ & $38(72 / 190)$ & $91^{*}(10 / 11)$ \\
TIA-1 & $32(62 / 195)$ & $46(6 / 13)$ \\
EBER & & $31(4 / 13)$ \\
$1-4+$ & $30(67 / 222)$ & $0(0 / 13)$ \\
\hline $3-4+$ & $14(30 / 222)$ & \\
\hline
\end{tabular}

${ }^{*} P<.05$ vs all other PTCL-NOS.

often expressed CD30. There were no significant immunophenotypic differences for cases that presented with extranodal disease only, or by geographic region.

\section{Diagnostic accuracy}

Two diagnoses were made by each of the 4 expert hematopathologists in each case based mainly on the histology, immunophenotype, and molecular genetic data (diagnosis 1), and with the additional complete clinical data (diagnosis 2). A consensus diagnosis of PTCL-NOS was reached in $71 \%$ of the cases by this review, and the agreement rate of diagnosis 2 from the experts with the consensus diagnosis of PTCL-NOS was $75 \%$. The most common disagreements with the consensus diagnosis were angioimmunoblastic T-cell lymphoma (34\%) and anaplastic large cell lymphoma, ALK-negative (13\%). However, the agreement rate of diagnosis 2 from the experts with the consensus diagnosis of lymphoepithelioid PTCL was only 58\%, with the most common disagreement being PTCL, unspecified (50\%).

A change in the diagnosis of PTCL-NOS (diagnosis 1) to the correct (consensus) diagnosis after consideration of the clinical data occurred in 154 cases, with 105 of these cases (68\%) changed to a diagnosis of adult T-cell leukemia/lymphoma with knowledge of the HTLV-1 status. Other common changes after consideration of the clinical data included angioimmunoblastic T-cell lymphoma (7\%) and enteropathy-associated T-cell lymphoma (11\%). Change from another diagnosis to the consensus diagnosis of PTCL-NOS also occurred in 14 cases, the most common being angioimmunoblastic T-cell lymphoma (43\%). The presence or absence of clinical features typical of angioimmunoblastic T-cell lymphoma, such as hypergammaglobulinemia, skin rash, autoantibodies, or autoimmune cytopenias, appeared to influence the diagnosis in borderline cases. All cases reclassified as entities other than PTCL-NOS were excluded from further analysis.

\section{Clinical features}

The clinical features of the 340 patients with PTCL-NOS are presented in Table 2. The median age for the patients was 60 years (range, 19-87 years), and the male-to-female ratio was 1.9:1. The majority of patients (69\%) had advanced stage (III/IV) disease. Only nodal disease was present in $38 \%$, nodal and extranodal disease in $49 \%$, and extranodal disease only was present in $13 \%$ of the patients. Hepatomegaly was noted in $17 \%$ and splenomegaly in $24 \%$ of the patients. Other common extranodal sites included the skin (16\%), subcutaneous tissue (6\%), and the lungs (8\%). A
Table 2. Clinical features of patients with PTCL-NOS

\begin{tabular}{lc}
\hline Characteristic & Value \\
\hline Median age, y & 60 \\
Male:female ratio & $1.9: 1$ \\
Stage, $\%(\mathrm{n} / \mathrm{N})$ & \\
\hline I & $14(45 / 334)$ \\
II & $17(57 / 334)$ \\
III & $26(87 / 334)$ \\
IV & $43(145 / 334)$ \\
B symptoms, $\%(\mathrm{n} / \mathrm{N})$ & $35(118 / 340)$ \\
Performance status $\geq 2, \%(\mathrm{n} / \mathrm{N})$ & $18(60 / 334)$ \\
Elevated serum LDH, \% (n/N) & $49(158 / 323)$ \\
Extranodal sites $>1, \%(\mathrm{n} / \mathrm{N})$ & $29(99 / 340)$ \\
Bone marrow positive, $\%(\mathrm{n} / \mathrm{N})$ & $21(68 / 322)$ \\
Bulky disease $\geq 10 \mathrm{~cm}, \%(\mathrm{n} / \mathrm{N})$ & $7(19 / 285)$ \\
Platelets $<150 \times 10 \% / \mathrm{L}, \%(\mathrm{n} / \mathrm{N})$ & $24(64 / 266)$ \\
Hypergammaglobulinemia, \% $(\mathrm{n} / \mathrm{N})$ & $14(29 / 201)$ \\
\hline
\end{tabular}

LDH indicates lactate dehydrogenase.

history of prior immunosuppressive therapy or immune system disorder was reported in 13 and 9 patients, respectively, and only one patient had HIV infection. Other findings included a hemoglobin of less than $110 \mathrm{~g} / \mathrm{L}(22 \%)$, circulating tumor cells $(10 \%)$, elevated serum $\beta_{2}$-microglobulin (36\%), C-reactive protein (50\%) or calcium (5\%) levels, hypogammaglobulinemia (9\%), monoclonal serum immunoglobulin (4\%), hemolytic anemia (3\%), and hemophagocytic syndrome (3\%).

\section{Treatment and outcome}

The median follow-up duration was 3.1 years, and the 5-year OS for the entire group was $32 \%$, whereas the 5-year (FFS) was only $20 \%$ (Figure 1). The OS at 1 and 3 years was $69 \%$ and $41 \%$, respectively, whereas the corresponding FFS was only $46 \%$ and $25 \%$, respectively. The majority of patients $(80 \%)$ received combination chemotherapy containing an anthracycline, whereas the rest received combination chemotherapy without an anthracycline (7\%), single-agent therapy (4\%), or no chemotherapy (9\%). The complete remission rate for those receiving an anthracyclinecontaining regimen was $56 \%$ and the 5 -year OS was $36 \%$, but the 5 -year FFS was only $22 \%$. There was no survival advantage for patients with PTCL-NOS who received combination chemotherapy containing an anthracycline compared with those receiving combination chemotherapy without an anthracycline. ${ }^{17}$ There were no differences in complete response rates or survival by geographic region. However, initial radiation therapy improved the OS of patients with stage 1 disease who also received chemotherapy

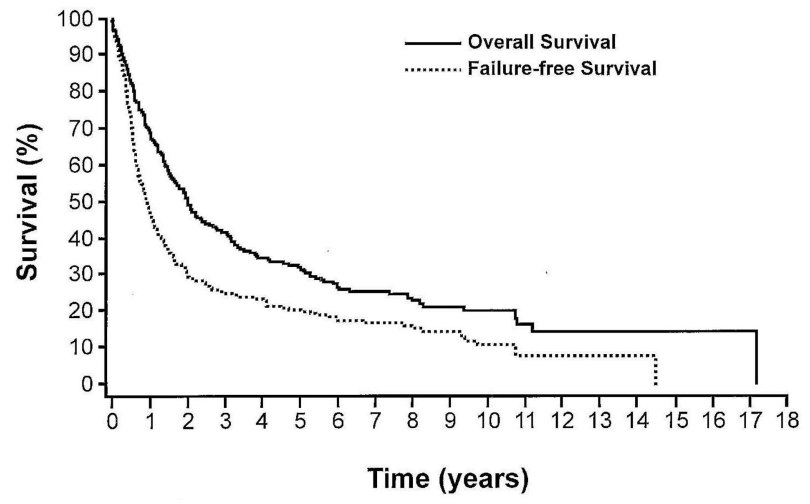

Figure 1. OS and FFS of 340 patients with PTCL-NOS. 


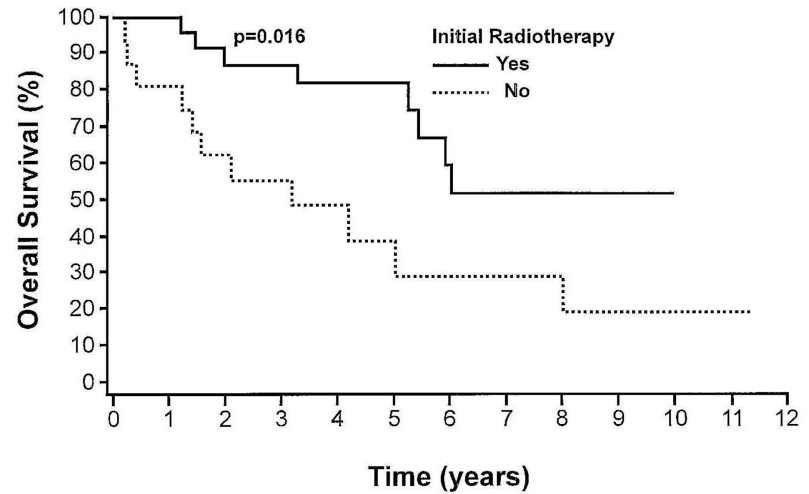

Figure 2. OS of patients with stage 1 PTCL-NOS who received initial radiation therapy in addition to chemotherapy $(n=25)$ compared with those who received only chemotherapy $(n=16)$.

( $\mathrm{n}=25)$ compared with those who received only chemotherapy $(\mathrm{n}=16$; Figure 2$)$. The result was similar for FFS $(P=.06)$. At the close of the study, $68 \%$ of the patients had died and only $9 \%$ were in remission at the time of death.

\section{Clinical prognostic factors}

All of the prognostic factors in the IPI were highly significant predictors of OS and FFS $(P<.001)$, and the IPI was predictive of both OS and FFS (Figure 3; Table 3). The more recently described prognostic index for PTCL-NOS (PIT), which includes 3 characteristics of the IPI (age, performance status, and lactate dehydrogenase level) and bone marrow involvement, was also predictive of survival (Figure 4; Table 3). However, bone marrow involvement was not a robust predictor of OS $(P=.03)$ or FFS $(P=.06)$ in our patients, and the PIT does not appear to be superior to the IPI in
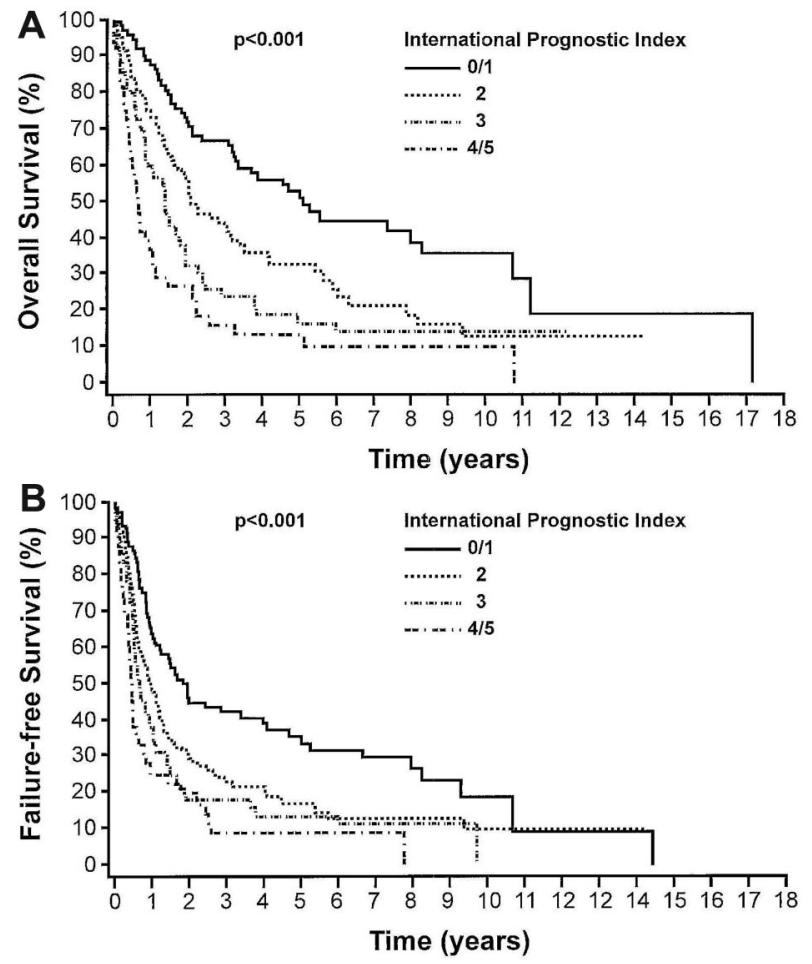

Figure 3. Survival. OS (A) and FFS (B) of patients with PTCL-NOS ( $n=315)$ according to the IPI.
Table 3. Survival of patients with PTCL-NOS by prognostic models

\begin{tabular}{lccc}
\hline $\begin{array}{l}\text { Model/no. of } \\
\text { risk factors }\end{array}$ & Cases, \% & 5-year OS, \% & 5-year FFS, \% \\
\hline IPI & & & \\
\hline $0 / 1$ & 28 & 50 & 36 \\
\hline 2 & 35 & 33 & 18 \\
\hline 3 & 22 & 16 & 15 \\
$4 / 5$ & 15 & 11 & 9 \\
\hline PIT & & & \\
\hline 0 & 20 & 50 & 34 \\
\hline 1 & 38 & 40 & 22 \\
\hline 2 & 29 & 22 & 13 \\
\hline $3 / 4$ & 13 & 11 & 8 \\
\hline
\end{tabular}

predicting the survival of patients with PTCL-NOS. Only one group with relatively good FFS was identified using either model.

We also evaluated other potential prognostic factors by univariate analysis and the following were adverse predictors of OS and FFS, respectively: B symptoms $(P=.004 ; P=.014)$, bulky disease $\geq 10 \mathrm{~cm}(P=.005 ; P=.004)$, elevated serum C-reactive protein $(P=.018 ; P=.008)$, circulating tumor cells $(P<.001$; $P<.001)$, and a platelet count of less than $150 \times 10^{9} / \mathrm{L}(P<.001$; $P<.001)$. The presence of hypergammaglobulinemia was a favorable prognostic indicator of OS and FFS $(P=.04 ; P=.03)$.

\section{Multivariate analysis of clinical prognostic factors}

By stepwise multivariate analysis, when controlling for the IPI, only bulky disease $\geq 10 \mathrm{~cm}$ was still predictive of survival, with a hazard ratio $(\mathrm{HR})$ of 2.1 for $\mathrm{OS}(P=.019)$ and 2.5 for FFS $(P=.003)$. A platelet count of less than $150 \times 10^{9} / \mathrm{L}$ was also predictive of FFS $(\mathrm{HR}=1.6, P=.016)$.
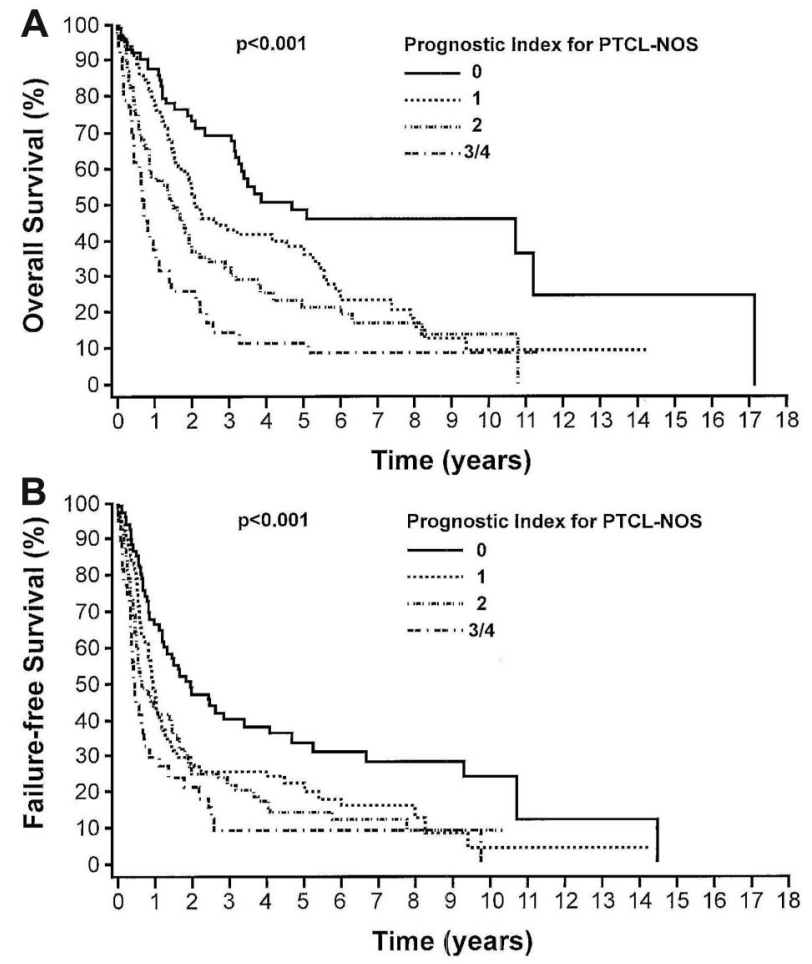

Figure 4. Survival. OS (A) and FFS (B) of patients with PTCL-NOS $(n=315)$ according to the PIT. 


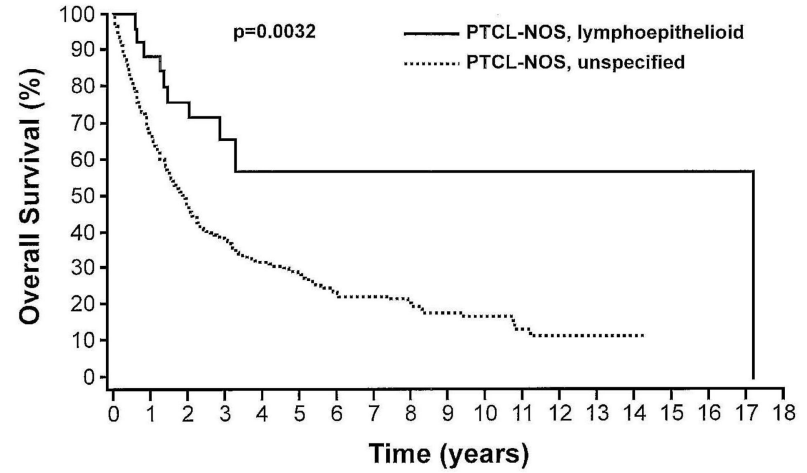

Figure 5. OS of patients with the lymphoepithelioid (Lennert) variant of PTCL-NOS $(n=28)$ compared with the unspecified cases of PTCL-NOS ( $\mathrm{n}=292$ ).

\section{Pathologic prognostic factors}

We also evaluated a variety of pathologic features as possible prognostic factors by univariate analysis, and the following were adverse predictors of OS and FFS, respectively: Ki67 proliferation more than $25 \%$ (75\% of cases; $P<.001 ; P=.009$ ), transformed tumor cells more than $70 \%(31 \% ; P=.008 ; P=.12)$, significant numbers of Epstein-Barr virus (EBV)-positive B cells (EBERs $3-4+, 14 \% ; P=.044 ; P=.48)$, and CD56 expression $(6 \%$; $P=.05 ; P=.04)$ and CD30 expression $(32 \% ; P=.053 ; P=.059)$ by more than $20 \%$ of the tumor cells. Interestingly, significant EBV positivity was predictive of adverse survival only in patients younger than 60 years $(P=.007 ; P=.18)$ but was not related to a history of immunosuppressive therapy or immune system disorder. The following pathologic features were favorable predictors of OS and FFS, respectively: lymphoepithelioid (Lennert) variant $(8.2 \% ; P=.003 ; P=.026$; Figure 5$)$ and background $\mathrm{CD}^{+}$ T cells more than $10 \%(23 \% ; P=.08 ; P=.005)$. There were no significant differences in the clinical prognostic factors between patients with the lymphoepithelioid (Lennert) variant and the others with PTCL-NOS (results not shown). Pathologic features that were not predictive of survival were the tumor T-cell phenotype $\left(\mathrm{CD} 4^{+}\right.$or $\mathrm{CD}^{+}$, or $\mathrm{CD}^{-}$and $\mathrm{CD} 8^{-}$) and a cytotoxic phenotype $\left(\mathrm{TIA}^{+}\right)$.

\section{Multivariate analysis of pathologic prognostic factors}

By stepwise multivariate analysis, when controlling for the IPI, only transformed tumor cells more than $70 \%$ were predictive of OS (HR $=1.7, P=.019)$, and no pathologic features were predictive of FFS. The expert pathologists were correct in their estimate of transformed tumor cells more than $70 \%$ in $88 \%$ of these cases.

\section{Final multivariate analysis of significant clinical and pathologic prognostic factors}

By stepwise multivariate analysis, when controlling for the IPI and considering only bulky disease $\geq 10 \mathrm{~cm}$, a platelet count less than $150 \times 10^{9} / \mathrm{L}$, and transformed tumor cells more than $70 \%$, only the latter was predictive of $\mathrm{OS}(\mathrm{HR}=2.2, P=.0002)$ and FFS $(\mathrm{HR}=1.6, P=.014)$.

\section{Discussion}

In our study of 1153 cases of PTCL and NKTCL, the 340 cases of PTCL-NOS represented $29.5 \%$ of all cases. This finding is in keeping with other smaller studies ${ }^{5-7,12,18-23}$ in which PTCL-NOS was usually the most frequent subtype, composing $17 \%$ to $59 \%$ of all cases. To date, our study is one of the largest reported series of PTCL-NOS. In our series, lymphoepithelioid (Lennert) PTCL was the most common special variant of PTCL-NOS, composing $2.4 \%$ of all cases and $8.2 \%$ of PTCL-NOS. This latter finding is in keeping with other studies of PTCL-NOS, 6,8,9,19 which have reported this variant in $2 \%$ to $13 \%$ of cases. The diagnostic accuracy of PTCL-NOS, that is the agreement rate of the expert hematopathologists with the consensus diagnosis, in our study was only $75 \%$, similar to that reported by others. ${ }^{6,12}$ Detailed clinical information was important for the correct diagnosis of PTCL-NOS, and particularly information on the HTLV-1 status of the cases from Japan. Without this latter information, lymph node biopsies of PTCL-NOS could not be reliably distinguished from adult T-cell leukemia/lymphoma. Because this disease also occurs in the West, HTLV-1 status should be evaluated and reported to the pathologist in patients at high risk for the disease or from endemic areas. Two other diagnoses, angioimmunoblastic T-cell lymphoma and enteropathy-associated T-cell lymphoma, were also sometimes clarified by the clinical information. These findings highlight some of the difficulties in the differential diagnosis of PTCL subtypes, particularly for cases at the border between different entities. For such cases, gene expression profiling may be very helpful in the future for confirming the correct diagnosis. ${ }^{24-27}$

The clinical features of our patients with PTCL-NOS are similar to those reported by other studies..$^{5-13,20,23,28}$ The median age of our patients was 60 years (range, 19-87 years), with a male predominance, and the majority of the patients $(69 \%)$ presented with advanced stage disease. Most of our patients (87\%) presented with nodal disease, but extranodal disease was also present in $49 \%$ of these patients, whereas only $13 \%$ presented with extranodal disease only. As in other studies, ${ }^{5-13,20-23,28}$ the majority of our patients $(80 \%)$ were treated with combination chemotherapy including an anthracycline, but the complete remission rate was low (56\%) and there were few cures, with no survival advantage for those receiving an anthracycline. The 5-year OS (32\%) and FFS (20\%) of the patients in our series were poor, but in keeping with the survival reported in other studies. . $^{5,20-23,28}$ The use of more intensified chemotherapy in patients with PTCL-NOS does not appear to be beneficial, ${ }^{7,11}$ although one study reported improved survival. ${ }^{12}$ Therefore, new treatment regimens are clearly needed for PTCLNOS, including the addition of novel agents to existing regimens, new regimens combining novel agents, use of monoclonal antibodies, and innovative strategies for stem cell transplantation..$^{29-32} \mathrm{We}$ did find that initial radiation therapy improved the survival of patients with stage 1 disease who also received chemotherapy compared with those who received only chemotherapy, similar to the experience with diffuse large B-cell lymphoma. ${ }^{33,34}$ However, our study was retrospective in nature and selection bias may have been present.

To apply and evaluate new treatment strategies for PTCL-NOS, reliable clinical and pathologic prognostic factors are needed for a rational and risk-adapted approach. Although a small number of studies $^{5,7-13}$ have attempted to identify the clinical features of prognostic importance in PTCL-NOS, these studies have generally consisted of small numbers of cases and the findings have been inconsistent or unconfirmed. In our study, all of the prognostic factors in the IPI were highly significant predictors of OS and FFS $(P<.001)$, and the IPI was predictive of both OS and FFS (Figure 3). However, only patients with an IPI score of 0 or 1 had a favorable FFS. These findings regarding the IPI have, 
in general, been confirmed by the other studies., ${ }^{5,-9,11-13}$ We also identified several other clinical prognostic factors by univariate analysis, but only bulky disease $(\geq 10 \mathrm{~cm})$ and thrombocytopenia $\left(<150 \times 10^{9} / \mathrm{L}\right)$ were independent predictors of survival when controlling for the IPI. However, other studies performing multivariate analysis with the IPI in PTCL-NOS did not evaluate these 2 clinical features, and no other consistent findings were reported. $5,9,11,13$ In another study, bulky disease dropped out in multivariate analysis. ${ }^{10}$ Therefore, we think that the IPI, along with bulky disease and thrombocytopenia, could be used to stratify patients with PTCL-NOS for novel and risk-adapted therapies.

In 2004, Gallamini et al proposed a new PIT that incorporated 3 parameters of the IPI: age more than 60 years, performance status $\geq 2$, and elevated serum lactate dehydrogenase, as well as bone marrow involvement. ${ }^{9}$ Although they found the IPI to be highly predictive of OS $(P<.001)$, they concluded that the PIT was superior to the IPI. One concern about the PIT is that the local diagnosis of record was used in development of the model and the diagnosis of PTCL-NOS was not confirmed by an expert hematopathologist. However, others have also found the PIT to be predictive of survival in PTCL-NOS. ${ }^{10,12,13}$ In our study, however, the PIT does not appear to be superior to the IPI (Figure 4, Table 3). Using a similar strategy, but with a small number of cases, Kojima et $\mathrm{al}^{8}$ and Went et $\mathrm{al}^{10}$ have also proposed new prognostic models for PTCL-NOS, but these models have not been validated by others.

We also evaluated a variety of pathologic features as possible prognostic factors in PTCL-NOS. In our study, Ki67 proliferation $(>25 \%)$ was an adverse predictor of survival. Ki67 proliferation ( $\geq 80 \%$ ) was also reported by Went et al to predict for poor survival and was incorporated into their prognostic model. ${ }^{10} \mathrm{We}$ also found that a high number of transformed tumor cells $(>70 \%)$ was predictive of poor survival, which has previously been reported by others. ${ }^{6,19}$ Dupuis et al have shown that the presence of EBV-encoded RNAs (EBERs) in the tumor tissue is an adverse predictor of survival in older patients ( $>60$ years) with PTCLNOS. ${ }^{35}$ In contrast, we found that significant EBV infection (EBERs $3-4^{+}$) was predictive of poor survival only in our younger patients $(<60$ years). Some studies have reported that a T-helper cell phenotype $\left(\mathrm{CD} 4^{+}, \mathrm{CD}^{-}\right)$predicts for better survival, but we could not confirm this finding. ${ }^{8,10}$ Asano et al have reported that a cytotoxic phenotype (ie, the expression of TIA-1 or granzyme B) is predictive of poor survival in PTCL-NOS, but EBV was also found in $51 \%$ of their cases with a cytotoxic phenotype. ${ }^{36} \mathrm{We}$ and others ${ }^{10,37}$ could not confirm that a cytotoxic phenotype is an adverse prognostic factor in PTCL-NOS. However, Iqbal et al, using gene expression profiling, recently identified a molecular subgroup among PTCL-NOS with features of cytotoxic lymphocytes and a poor survival. ${ }^{26}$ Additional studies are needed to clarify these discrepant findings in PTCL-NOS.

We also found 2 pathologic features that were favorable predictors of survival in PTCL-NOS, a high background of reactive $\mathrm{CD}^{+} \mathrm{T}$ cells $(>10 \%)$ and the lymphoepithelioid (Lennert) variant of PTCL-NOS, which is characterized by a high content of epithelioid histocytes in clusters. These findings suggest that the tumor microenvironment may play an important role in the biology of some cases of PTCL-NOS. The excellent survival of our cases of lymphoepithelioid lymphoma could not be explained by other clinical prognostic factors, although these cases were generally characterized by atypical small lymphoid cells with few transformed tumor cells admixed. However, others ${ }^{38,39}$ have reported a poor survival for patients with lymphoepithelioid lymphoma. The characteristic morphology and phenotype of lymphoepithelioid lymphoma, which is often derived from $\mathrm{CD}^{+}$cytotoxic $\mathrm{T}$ cells, ${ }^{37}$ as well as the good survival suggest that this special variant of PTCL-NOS should be further studied and possibly separated as a distinctive entity in future lymphoma classifications.

Multivariate analysis of the pathologic prognostic factors was also performed and, when controlling for the IPI, only the number of transformed tumor cells $(>70 \%)$ was predictive of survival. In a final multivariate analysis of the significant clinical and pathologic prognostic factors, when controlling for the IPI, only the number of transformed tumor cells remained predictive of OS and FFS. This feature also appears to be reproducible because the expert pathologists were correct in their estimate of transformed tumor cells more than $70 \%$ in $88 \%$ of the cases. Thus, the IPI and this one simple and easy to evaluate pathologic feature could be used to stratify patients with PTCL-NOS for novel and risk-adapted therapies.

As our knowledge of the molecular and genetic features of PTCL evolves in the future, new entities within the heterogeneous category of PTCL-NOS will be discovered. For example, the recent study of Iqbal et al, using gene expression profiling, identified a molecular subgroup with the features of cytotoxic T cells and a poor outcome compared with other cases of PTCL-NOS. ${ }^{26} \mathrm{New}$ and better molecular and genetic prognosticators will also be developed for the various PTCL entities, such as that recently reported for angioimmunoblastic T-cell lymphoma. ${ }^{26}$ Hopefully, new discoveries will also lead to more rational and effective targeted therapies for the various PTCL entities in the future.

\section{Acknowledgments}

The authors thank Martin Bast and Fred Ullrich for the data collection and analysis, and Ventana Medical Systems Inc (Tucson, AZ) for donation of the use of a Ventana XT immunostainer for the project.

A complete list of study sites and physicians participating in the International Peripheral T-cell Lymphoma Project appears in the supplemental Appendix.

\section{Authorship}

Contribution: D.D.W. designed the research, collected the data, contributed material and reagents, participated in the pathology review, analyzed and interpreted the data, and wrote the manuscript; K.J.S. collected and interpreted the data; N.L.H., R.D.G., and E.S.J. contributed material, participated in the pathology review, and interpreted the data; K.A.M., T.R., S.P., and S.N. contributed material and reagents, collected data, performed research, and participated in the pathology review; B.N., E.C., and F.B. contributed material and participated in the pathology review; B.C., W.-S.K., H.H., M.F., W.Y.A., and K.T. collected and interpreted the data; and J.O.A. and J.M.V. designed the research and collected, analyzed, and interpreted the data.

Conflict-of-interest disclosure: The authors declare no competing financial interests.

Correspondence: Dennis D. Weisenburger, Department of Pathology and Microbiology, University of Nebraska Medical Center, 983135 Nebraska Medical Center, Omaha, NE 68198-3135; email:dweisenb@unmc.edu. 


\section{References}

1. Swerdlow SH, Campo E, Harris NL, et al, eds. WHO Classification of Tumours of Haematopoietic and Lymphoid Tissues. Lyon, France: International Agency for Research on Cancer; 2008.

2. Anderson JR, Armitage JO, Weisenburger DD. Epidemiology of the non-Hodgkin's lymphomas: distributions of the major subtypes differ by geographic locations. Ann Oncol. 1998;9(7):717-720.

3. Morton LM, Wang SS, Devesa SS, Hartge P, Weisenburger DD, Linet MS. Lymphoma incidence pattern by WHO subtype in the United States, 1992-2001. Blood. 2006;107(1):265-276.

4. Abouyabis AN, Shenoy PJ, Lechowicz NJ, Flowers CR. Incidence and outcomes of peripheral T-cell lymphoma subtypes in the United States. Leuk Lymphoma. 2008;49(11):2099 2107

5. Lopez-Guillermo A, Cid J, Salar A, et al. Peripheral T-cell lymphomas: initial features, natural history, and prognostic factors in a series of $174 \mathrm{pa}$ tients diagnosed according to the REAL classification. Ann Oncol. 1998;9(8):849-855.

6. Rüdiger T, Weisenburger DD, Anderson JR, et al. Peripheral T-cell lymphoma (excluding anaplastic large-cell lymphoma): results from the Non-Hodgkin's Lymphoma Classification Project. Ann Oncol. 2002;13(1):140-149.

7. Savage KJ, Chhanabhai M, Gascoyne RD, Connors JM. Characterization of peripheral T-cell lymphomas in a single North American institution by the WHO classification. Ann Oncol. 2004 15(10):1467-1475.

8. Kojima H, Hasegawa Y, Suzukawa K, et al. Clinicopathological features and prognostic factors of Japanese patients with "peripheral T-cell lymphoma, unspecified" diagnosed according to the WHO classification. Leuk Res. 2004;28(12):12871292.

9. Gallamini A, Stelitano C, Calvi R, et al. Peripheral T-cell lymphoma unspecified (PTCL-U): a new prognostic model from a retrospective multicentric clinical study. Blood. 2004;103(7):2474-2479.

10. Went $P$, Agostinelli $C$, Gallamini A, et al. Marker expression in peripheral T-cell lymphoma: a proposed clinical-pathologic prognostic score. J Clin Oncol. 2006;24(16):2472-2479.

11. Prochazka V, Trneny M, Pytlik R, et al. Peripheral T-cell lymphoma, unspecified: the analysis of the data from the Czech Lymphoma Study Group (CLSG) registry. Biomed Pap Med Fac Univ Palacky Olomouc Czech Repub. 2007;151(1): 103-107.

12. Niitsu N, Okamoto M, Nakamine H, Aoki S, Motomura S, Hirano M. Clinico-pathologic features and outcome of Japanese patients with peripheral T-cell lymphomas. Hematol Oncol. 2008; 26(3):152-158.

13. Lee Y, Uhm JE, Lee HY, et al. Clinical features and prognostic factors of patients with "peripheral T-cell lymphoma, unspecified." Ann Hematol. 2009;88(2):111-119.

14. International Non-Hodgkin's Lymphoma Prognostic Factors Project. A predictive model for aggressive non-Hodgkin's lymphoma: the International Non-Hodgkin's Lymphoma Prognostic Factors Project. N Engl J Med. 1993;329(14):987-994.

15. Kaplan EL, Meier P. Nonparametric estimation from incomplete observations. J Am Stat Assoc. 1958;53(282):457-481.

16. Cox DR. Regression models and life tables. $J R$ Stat Soc B. 1972;34(2):187-220.

17. Vose J, Armitage J, Weisenburger D. International Peripheral T-cell and Natural Killer/T-Cell Lymphoma Study: pathology findings and clinical outcomes. J Clin Oncol. 2008;26(25):4124-4130.

18. Non-Hodgkin's Lymphoma Classification Project. A clinical evaluation of the International Lymphoma Study Group classification of non-Hodgkin's lymphoma. Blood. 1997;89(11):3909-3918.

19. Ascani S, Zinzani PL, Gherlinzoni F, et al. Peripheral T-cell lymphomas: Clinico-pathologic study of 168 cases according to the REAL classification. Ann Oncol. 1997;8(6):583-592.

20. Pellatt J, Sweetenham J, Pickering RM, Brown L, Wilkins B. A single-centre study of treatment outcomes and survival in 120 patients with peripheral T-cell non-Hodgkin's lymphoma. Ann Hematol. 2002;81(5):267-272.

21. Kim K, Kim WS, Jung CW, et al. Clinical features of peripheral T-cell lymphomas in 78 patients diagnosed according to the Revised EuropeanAmerican Lymphoma (REAL) classification. Eur J Cancer. 2002;38(1):75-81.

22. Arrowsmith ER, Macon WR, Kinney MC, et al. Peripheral T-cell lymphomas: clinical features and prognostic factors of 92 cases defined by the Revised European-American Lymphoma classification. Leuk Lymphoma. 2003;44(2):241-249.

23. Au WY, Ma SY, Chim CS, et al. Clinicopathologic features and treatment outcome of mature T-cell and natural killer-cell lymphomas diagnosed according to the World Health Organization classification scheme: a single center experience of 10 years. Ann Oncol. 2005;16(2):206-214.

24. de Laval L, Bisig B, Thielen C, Boniver J, Gaulard P. Molecular classification of T-cell lymphomas. Crit Rev Oncol Hematol. 2009;72(2):125-143.

25. Costello R, Sanchez C, Le Treut T, Rihet $P$, Imbert J, Sebahoun G. Peripheral T-cell lymphoma gene expression profiling and potential therapeutic exploitations. $\mathrm{Br} J$ Haematol 2010; 150(1):21-27.

26. Iqbal J, Weisenburger DD, Greiner TC, et al. Molecular signatures to improve diagnosis in peripheral T-cell lymphoma and prognostication in an- gioimmunoblastic T-cell lymphoma. Blood. 2010; 115(5):1026-1036

27. Piva R, Agnelli L, Pellegrino E, et al. Gene expression profiling uncovers molecular classifiers for the recognition of anaplastic large-cell lymphoma within peripheral T-cell neoplasms. J Clin Oncol. 2010;28(9):1583-1590.

28. Sonnen R, Schmidt WP, Müller-Hermelink HK, Schmitz N. The International Prognostic Index determines the outcome of patients with nodal mature T-cell lymphomas. Br J Haematol. 2005; 129(3):366-372

29. Rodriguez J, Gutierrez A, Martinez-Delgado B, Perez-Manga $G$. Current and future aggressive peripheral T-cell lymphoma treatment paradigms, biological features and therapeutic molecular targets. Crit Rev Oncol Hematol. 2009;71(3):181198.

30. Zain JM, O'Connor O. Targeted treatment and new agents in peripheral T-cell lymphoma. Int J Hematol. 2010;92(1):33-44.

31. Foss FM. Enhancing existing approaches to peripheral T-cell lymphoma. Semin Hematol. 2010; 47(suppl 1):S8-S10.

32. Zinzani PL. High-dose therapy and stem cell transplantation. Semin Hematol. 2010;47(suppl 1):S15-S17.

33. Persky DO, Miller TP. Localized large cell lymphoma: is there any need for radiation therapy? Curr Opin Oncol. 2009;21(5):401-406.

34. Phan J, Mazloom A, Medeiros LJ, et al. Benefit of consolidative radiation therapy in patients with diffuse large B-cell lymphoma treated with R-CHOP chemotherapy. J Clin Oncol. 2010; 28(27):4170-4176.

35. Dupuis J, Emile JF, Mounier N, et al. Prognostic significance of Epstein-Barr virus in nodal peripheral T-cell lymphoma, unspecified: a Group d'Etude des Lymphomes de l'Adulte (GELA) study. Blood. 2006;108(13):4163-4169.

36. Asano N, Suzuki R, Kagami Y, et al. Clinicopathologic and prognostic significance of cytotoxic molecule expression in nodal peripheral T-cell lymphoma, unspecified. Am J Surg Pathol. 2005; 29(10):1284-1293.

37. Geissinger E, Odenwald T, Lee SS, et al. Nodal peripheral T-cell lymphomas and, in particular, their lymphoepithelioid (Lennert's) variant are often derived from CD8+ cytotoxic T cells. Virchows Arch. 2004;445(4):334-343.

38. Patsouris $\mathrm{E}$, Engelhard M, Zwingers $\mathrm{T}$, Lennert $\mathrm{K}$. Lymphoepithelioid cell lymphoma (Lennert's lymphoma): clinical features derived from analysis of 108 cases. Br J Haematol. 1993;84(2):346-348.

39. Gisselbrecht C, Gaulard P, Lepage E, et al. Prognostic significance of T-cell phenotype in aggressive non-Hodgkin's lymphomas. Blood. 1998;92(1): 76-82. 


\section{( ) blood}

2011 117: 3402-3408

doi:10.1182/blood-2010-09-310342 originally published online January 26, 2011

\section{Peripheral T-cell lymphoma, not otherwise specified: a report of $\mathbf{3 4 0}$ cases from the International Peripheral T-cell Lymphoma Project}

Dennis D. Weisenburger, Kerry J. Savage, Nancy Lee Harris, Randy D. Gascoyne, Elaine S. Jaffe, Kenneth A. MacLennan, Thomas Rüdiger, Stefano Pileri, Shigeo Nakamura, Bharat Nathwani, Elias Campo, Francoise Berger, Bertrand Coiffier, Won-Seog Kim, Harald Holte, Massimo Federico, Wing Y. Au, Kensei Tobinai, James O. Armitage, Julie M. Vose and for the International Peripheral T-cell Lymphoma Project

Updated information and services can be found at:

http://www.bloodjournal.org/content/117/12/3402.full.html

Articles on similar topics can be found in the following Blood collections

Clinical Trials and Observations (4213 articles)

Free Research Articles (3487 articles)

Lymphoid Neoplasia (2168 articles)

Information about reproducing this article in parts or in its entirety may be found online at: http://www.bloodjournal.org/site/misc/rights.xhtml\#repub_requests

Information about ordering reprints may be found online at:

http://www.bloodjournal.org/site/misc/rights.xhtml\#reprints

Information about subscriptions and ASH membership may be found online at:

http://www.bloodjournal.org/site/subscriptions/index.xhtml

Blood (print ISSN 0006-4971, online ISSN 1528-0020), is published weekly by the American Society of Hematology, 2021 L St, NW, Suite 900, Washington DC 20036.

Copyright 2011 by The American Society of Hematology; all rights reserved. 